\title{
Qualität in der Integrierten Versorgung
}

\author{
Über Nutzen und Qualität der Integrierten Versorgung wird kontrovers und \\ vorwiegend spekulativ diskutiert. Wenn die jüngst publizierte Studie zu Qualitäts- \\ assessments jetzt eine signifikante Effektivität nachweist und die weit über- \\ wiegende Zahl der Ärztinnen und Ärzte, die sich konsequenten Qualitätsnach- \\ weisen unterziehen, in Netzwerken arbeitet, so sind dies ermutigende Signale für \\ die Integrierte Versorgung.
}

Kurt Hess

Korrespondenz:

Dr. med. et

Dipl. Ing. ETH Kurt Hess

Sophienstrasse 2

CH-8032 Zürich

kurt.hess[at]hin.ch

\begin{abstract}
Gleich mehrere Artikel des KVG [1] regeln wesentliche Aspekte der Qualitätssicherung, doch umgesetzt ist davon insbesondere im ambulanten Bereich noch wenig. Qualitätsassessments in Arztpraxen und unabhängige Qualitätsnachweise - etwa im Sinne von Zertifikationen - haben in der Ärzteschaft auch heute noch einen schweren Stand. Geltend gemacht werden meist ein unverhältnismässiger Aufwand, das Fehlen geeigneter Qualitätskriterien und - wie sollte es anders sein - die nicht vorhandenen finanziellen Anreize. Und im Besonderen wird immer darauf hingewiesen, dass der Nachweis einer positiven Wirkung von Qualitätsassessments nicht erbracht ist. Auch die von den diversen Systemen postulierte Effektivität von Qualitätsassessments basierte bis dahin mehr auf anekdotischen als systematischen Erkenntnissen.
\end{abstract}

\section{Welche Wirkung hat Qualitätsmanagement in Arztpraxen?}

Erstmals ist nun Ende 2011 eine methodisch aufwendige Evaluationsstudie publiziert worden, die sich mit dieser Materie befasst [2]. Sie basiert auf dem System des bekannten European Practice Assessment (EPA). Insgesamt haben dabei 102 Praxen als Interventionsgruppe das komplette EPA-Verfahren zwei-

\section{Tabelle 1}

182 EPA-Indikatoren, gegliedert in fünf sogenannte Domänen.

\section{EPA-Domäne Thematik / Hauptindikatoren}

Qualität und Sicherheit Prüfung der Ausstattung, Infektions- und Verletzungsschutz, CIRS, (35 Indikatoren) Beschwerdemanagement, Teamarbeit, Qualitätsziele und -massnahmen

Information (45) KG-Führung, Patienteninformation, Datenschutz, Fachliteratur, Wahrung der Intimsphäre

Infrastruktur (38) Praxiserreichbarkeit, Verfügbarkeit, medizinische und nicht-medizinische Ausstattung

Menschen (62) Patientenbefragung nach Europep, Teamzufriedenheit

Finanzen (6) Finanzplanung, Budgetierung, Bilanz, Erfolgsrechnung

\section{La qualité dans les soins intégrés}

Les discussions sur l'utilité et la qualité des soins intégrés suscitent controverses et spéculations. La dernière étude en date sur les évaluations de la qualité démontre toutefois que les réseaux sont d'une grande efficacité. La majorité des médecins qui se soumettent régulièrement à des évaluations de la qualité travaillent en réseau. Des résultats encourageants pour les soins intégrés.

mal im Abstand von rund drei Jahren durchlaufen. Untersucht wurden in der Befragung zu allen EPAIndikatoren einerseits die Differenzen zwischen dem ersten und dem zweiten Assessment sowie andererseits die Unterschiede zu einer Kontrollgruppe von 209 Praxen.

Das EPA-System ist in den Jahren 2001 bis 2005 mit Unterstützung der Bertelsmann Stiftung durch eine renommierte internationale Expertengruppe für Hausarztpraxen entwickelt worden. Sämtliche Qualitätsindikatoren wurden systematisch auf Qualitätsrelevanz und Praktikabilität validiert. Damit ist ein weithin beachtetes, mehrfach prämiertes und laufend weiterentwickeltes QualitätsmanagementSystem entstanden, das heute im Bereich der ambulanten Grundversorgung wohl die Standards setzt.

Die 182 EPA-Indikatoren werden in fünf sogenannte Domänen gegliedert (Tab. 1).

Die Studienergebnisse sind ermutigend. In allen fünf Domänen des EPA-Indikatorensets werden in der erwähnten Studie signifikante Verbesserungen zwischen dem ersten und dem Re-Assessment wie auch gegenüber der Vergleichsgruppe nachgewiesen. 
Tabelle 2

Veränderungen Erstassessment ( $\left.T_{0}\right)$ vs. Re-Assessment nach 3 Jahren $\left(T_{1}\right)$ sowie Interventions- vs. Kontrollgruppe.

\begin{tabular}{|c|c|c|c|c|c|}
\hline EPA-Domäne & $\begin{array}{l}\text { Intervention } \mathrm{T}_{0} \\
\%^{*}\end{array}$ & $\begin{array}{l}\text { Intervention } \mathrm{T}_{1} \\
\%^{*}\end{array}$ & $\begin{array}{l}\Delta \mathrm{T}_{0}=>\mathrm{T}_{1} \\
\% * *\end{array}$ & $\begin{array}{l}\text { Kontrollgruppe } \\
\%^{*}\end{array}$ & $\begin{array}{l}\Delta \mathrm{T}_{1} \text { vs. Kontrollgruppe } \\
\% * *\end{array}$ \\
\hline Qualität und Sicherheit & 72,3 & 82,4 & 10,1 & 73,9 & 8,5 \\
\hline Information & 80,1 & 85,8 & 5,7 & 81,1 & 4,7 \\
\hline Infrastruktur & 74,9 & 84,9 & 10,0 & 77,8 & 7,1 \\
\hline Menschen & 73,9 & 79,0 & 5,1 & 75,7 & 3,3 \\
\hline Finanzen & 82,8 & 86,9 & 4,1 & 79,1 & 7,8 \\
\hline Gesamt & 75,2 & 82,6 & 7,4 & 77,7 & 4,9 \\
\hline
\end{tabular}

Die Zielerreichungsgrade bei den einzelnen Domänen ergeben sich aus den Mittelwerten der zugrundeliegenden Indikatoren. Die Differenzen $\mathrm{T}_{0}=>\mathrm{T}_{1}$ und $\mathrm{T}_{1}$ vs. Kontrollgruppe sind für jeden einzelnen der 182 EPA-Indikatoren ermittelt worden (Tab. 2).

Die prägnantesten Verbesserungen vom ersten zum zweiten Assessment werden nachgewiesen in

- Aus- und Weiterbildung (33,0\%),

- Beschwerdemanagement (29,7\%),

- Medizinischer Ausstattung (16,0\%),

- Prävention (15,2\%),

- Qualitätsentwicklung (14,9\%),

- Personalmanagement (13,4\%).

\section{Die Qualitätssysteme für die ambulante Grundversorgung}

In der Schweiz werden vier bis fünf Systeme für Qualitätsnachweise in der Grundversorgung angeboten [3]. Die Stringenz ist höchst unterschiedlich und reicht von blosser Selbstdeklaration bis zu konsequenten Vor-Ort-Visitationen mit schweiz- und teils europaweitem Online-Benchmarking.

Zur letzteren Kategorie gehört das erwähnte EPASystem, das in der Schweiz von EQUAM [4] in einer engen Kooperation mit den EPA-Autoren angeboten wird. Das Instrumentarium von EQUAM ist modular kreditierungsstelle (SAS/seco) für die Zertifizierung aller drei Module akkreditiert und unterzieht sich damit ihrerseits regelmässig einer konsequenten Qualitätssicherung. Der Zertifizierer erfüllt auch die anspruchsvollen Empfehlungen der SAMW [6]. Die detaillierten EPA-Indikatoren mit den Zertifizierungsanforderungen finden sich unter www.equam. ch in den Quicklinks.

\section{Welche Fraktionen der Ärzteschaft setzen systematische Qualitätsassessments ein?}

Die Untersuchung zeigt, dass in keiner Kategorie der Versorgungssysteme das Qualitätsmanagement so systematisch betrieben wird wie in der Integrierten Versorgung. Allein von den 346 durch EQUAM zertifizierten Ärzten arbeiten 307 (89\%) in gutstrukturierten Netzwerken. Auch einige der nicht auf EQUAM ausgerichteten grösseren Ärztenetze betreiben zumeist ein Qualitätsmanagement, allerdings mit unterschiedlichem Anspruchsniveau und teils auf der Basis einer Selbstdeklaration. So hat das grosse Ärztenetz Argomed mit rund 700 Ärztinnen und Ärzten unter dem Titel «Mehrfacharzt» ein umfangreiches Qualitätsinstrument entwickelt, das bis dahin aber erst von wenigen Netzmitgliedern in Pilotassessments genutzt worden ist. Auch Sanacare

\section{Die Untersuchung zeigt, dass nirgends das Qualitätsmanagement so systematisch betrieben wird wie in der Integrierten Versorgung.}

aufgebaut und bildet die Grundlage zur Qualitätszertifizierung von Ärzten und Praxen (Modul A auf EPABasis) sowie von Ärztenetzwerken (Modul B für integrierte Versorgung). Als Innovation entwickelt EQUAM zudem seit 2008 ein Instrument, das die Ergebnis-, Indikations- und Performancequalität von bis dahin drei Diagnosen zertifiziert (optionales Modul C) [5]. EQUAM ist von der Schweizerischen Ak- und Swica praktizieren für ihre HMO-Praxen Qualitätskontrollen unterschiedlicher Stringenz. Es kann damit als sicher gelten, dass nur eine kleine Minderheit der nicht in Netzen organisierten Ärztinnen und Ärzten sich einem der etablierten Qualitätsnachweise unterziehen.

Die Statistik zeigt, dass sich in der Schweiz über 50\% aller Allgemeinärzte und mehr als 400 andere 
spezialisierte Fachärzte einem der 86 Ärztenetzwerke angeschlossen haben. Es gilt allerdings zu beachten, dass der Begriff Ärztenetz nicht fest definiert ist, sondern derzeit noch von hochstrukturierten Netzwerken bis hinunter zu unverbindlichen Interessengemeinschaften reicht, die von gewissen Versicherern recht salopp als Ärztenetz bezeichnet werden. Ein lockeres Gatekeeping kann nicht als Kriterium für Ärztenetze genügen. Die Managed-Care-Vorlage strebt hier klarere Definitionen und Abgrenzungen an. Immerhin wächst die innere Verbindlichkeit der Netze kontinuierlich an, was sich u. a. darin zeigt, dass 84\% der Netzwerke mit den Krankenversicherern mindestens eine gewisse Budgetmitverantwortung vertraglich vereinbart haben.

\section{Was bedeuten diese Facts für die Integrierte Versorgung?}

Ein konsequentes Qualitätsmanagement mit systematischen Assessments weist eine signifikante qualitätsrelevante Wirkung aus. Es darf vermutet werden, dass dies speziell für professionelle und auf die Nachhaltigkeit ausgerichtete Qualitätssysteme gilt. Der überwiegende Teil der Ärzteschaft, der sich systematischen Nachweisen unterzieht, ist in Modellen der Integrierten Versorgung organisiert. Auch hier gilt die Vermutung, dass es sich wohl überwiegend um die gutstrukturierten und ambitionierten Netzwerke handelt. Und schliesslich nehmen wir zur Kenntnis, dass bereits über die Hälfte der schweizerischen Grundversorger in Modellen der Integrierten Versorgung organisiert ist. Und hier darf wohl zu Recht vermutet werden, dass die lockeren Netze auch ein eher lockeres Qualitätsmanagement praktizieren.

So weit die Facts, die - bewusst übervorsichtig interpretiert - dafür votieren, dass die Integrierte Versorgung zumindest die medizinische Qualität der
Standardversorgung erreicht und sie zudem permanent verbessert. Dies ist aber in der Tat sehr zurückhaltend formuliert. Es gilt ja dann wohl auch das Argument, dass die Koordination und Abstimmung der diversen Behandlungsschritte innerhalb und über die eigene Praxis hinaus doch wohl kaum qualitätslimitierend wirken dürften.

Oder um einiges realitätsbezogener und als Kontrapunkt zum angedrohten Verlust der freien Arztwahl: Integrierte Versorgung ist die freie Wahl von sorgfältig und auch nach qualitätsrelevanten Kriterien ausgewählten Ärztinnen und Ärzten.

\section{Literatur}

1 KVG Art. 22a, Art. 58, Art. 77.

2 Szecsenyi J, Campbell S, Broge B, Laux G, Willms S, Wensing $\mathrm{M}$ et al. Effectiveness of a quality-improvement program in improving management of primary care practices. CMAJ. vol. 183 no. 18; 2011. doi: 10.1503/cmaj.110412

Götz K, Szecsenyi J, Broge B, Willms S. Welche Wirkung hat Qualitätsmanagement in Arztpraxen? Göttingen: AQUA-Institut für angewandte Qualitätsförderung; 2011.

3 EQUAM - Externe Qualitätssicherung in der Medizin; QMN - Quali-med-net von medswiss.net; Mehrfacharzt von Argomed; Good Medical Practice von SQS; im status nascendi: VEDAG - Verband Deutschschweizer Ärztegesellschaften.

4 EQUAM - Externe Qualitätssicherung in der Medizin; vom Bund (seco) akkreditierte unabhängige Stiftung zur Zertifizierung von Ärzten, Praxen und Ärztenetzen der Grundversorgung. www.equam.ch

5 Hypertonie, Diabetes mellitus, koronare Herzkrankheit (Letzteres wieder in Kooperation mit EPA entwickelt).

6 Schweizerische Akademie der Medizinischen Wissenschaften. Zertifizierung im medizinischen Kontext. Basel; Juni 2011. 